\title{
Der Klimawandel betrifft auch die Gesundheit
}

\author{
Damiano Urbinelloa, Nino Künzlib \\ a Dr. phil. nat., Bundesamt für Gesundheit (BAG), Direktionsbereich Gesundheitspolitik; \\ ${ }^{b}$ Prof. Dr. med. et PhD, Vizedirektor Schweizerisches Tropen- und Public Health-Institut und Direktor der Swiss School of Public Health (SSPH+)
}

Lancet benennt den Klimawandel als möglicherweise grösste globale Bedrohung des 21. Jahrhunderts [1]*. Die Veränderungen haben Auswirkungen auf Umwelt, Wirtschaft und Gesellschaft und stellen global ein erhebliches Risiko für die Gesundheit dar. Eine Planung und Umsetzung von Klimaanpassungsmassnahmen sind bereits heute erforderlich. Der daraus resultierende Nutzen für die Gesundheit wird Kernthema der Swiss Public Health Conference sein.

Das Klima wird sich längerfristig weiter verändern. Bis zum Ende des 21. Jahrhunderts ist auf der Basis von Klimamodellen eine globale Erwärmung, je nach Szenario und Wirksamkeit der klimapolitischen Massnahmen, von bis zu $4,8^{\circ} \mathrm{C}$ zu erwarten. Diese Temperaturzunahmen sind mit gesundheitlichen Risiken verbunden, was durch eine Vielzahl von Studien belegt wird. Extreme Wetterereignisse wie Hitzewellen stellen bereits heute eine Gefahr für die Gesundheit dar, wie das Beispiel des Sommers 2003 aufzeigt. Klimaprognosen deuten beispielsweise darauf hin, dass Hitzeepisoden in Zukunft häufiger und intensiver auftreten.

Der Hitzesommer 2003 war in Europa einer der heissesten der letzten fünf Jahrhunderte. Die Temperaturen lagen in der Schweiz und in grossen Teilen Mitteleuropas zwischen 3 und $5^{\circ} \mathrm{C}$ über dem langjährigen Mittelwert [2]. Statistische Abschätzungen zeigen, dass in Gesamteuropa eine Exzess-Mortalität von rund 70000 Personen verzeichnet wurde [3]. In der Schweiz wurde eine Zusatzsterblichkeit von rund 975 Personen geschätzt, was einem Anteil von 7\% der mittleren Mortalitätsrate für die Monate Juni bis August 2003 entspricht [4]. Hitze kann die körperliche und geistige Leistungsfähigkeit deutlich reduzieren. Direkte Auswirkungen der Hitze sind beispielsweise Hitzeschlag, Dehydrierung, Bewusstlosigkeit, Hitzekrämpfe und Hyperthermie [5]. Insbesondere für alte und (chronisch) kranke Personen sowie Kleinkinder stellen längere Hitzeperioden ein lebensbedrohliches Risiko dar, weil diese vulnerablen Gruppen über eine schlechtere Thermoregulation verfügen. Sie schwitzen weniger und haben ein vermindertes Durstgefühl, was Herz und Kreislauf beeinflussen kann. Diverse Studien lie- fern zudem Hinweise auf eine erhöhte Anzahl Notfalleinweisungen aufgrund überwiegend respiratorischer Beschwerden bei lang andauernder Hitze. So nahmen z.B. in amerikanischen Städten die hitzebedingten Notfalleinweisungen um rund 3\% zu [6]. Ein ähnliches Bild wurde auch in europäischen Städten beobachtet [7]. Die Kombination von hohen Tagestemperaturen, fehlender nächtlicher Abkühlung und hoher Luftfeuchtigkeit dürfte ein entscheidender Erklärungsfaktor dafür sein.

Einkommensschwache Länder sind stärker vom Klimawandel betroffen und verfügen kaum über genügende Ressourcen für gezielte Vorsorgemassnahmen.

Das Bundesamt für Gesundheit hat zusammen mit dem Bundesamt für Umwelt in der Folge des Hitzesommers 2003 Informationsmaterial und Verhaltensempfehlungen für gefährdete Personen, deren Angehörige, Pflegepersonal und Ärzteschaft erarbeitet [8]. Zudem haben diverse Kantone Präventionsmassnahmen wie etwa Hitze-Frühwarnsysteme implementiert, um den Gesundheitsschutz der Bevölkerung zu gewährleisten. Wie das Beispiel Frankreich zeigt, können Präventionsstrategien die Folgen von Hitzewellen sehr erfolgreich einschränken. So konnte die hitzebedingte Mortalität während der Hitzewelle im Jahre 2006 dank integrierten Hitze-Notfallplänen deutlich reduziert werden. So wurde bei einer Annahme, dass sich die hitzebedingte Mortalität seit 2003 nicht geändert hat, eine ExzessMortalität von 6452 Fällen geschätzt. Es wurden 2065 hitzebedingte Todesfälle registriert Somit konnten auf der Basis dieser Präventionsmassnahmen ca. 4400 Todesfälle verhindert werden [9]. 
Der Klimawandel beeinflusst auch die zoonotischen Transmissionsmechanismen und folglich das Auftreten von Vektor-übertragenen Infektionskrankheiten [10]. Die Aedes albopictus (Asiatische Tigermücke) hat sich in Folge des globalen Handels inzwischen in Südeuropa etabliert, wo sie sich nun an die tieferen Temperaturen adaptiert. Mit den zukünftigen veränderten klimatischen Bedingungen und projizierten Temperaturzunahmen ist mit einer Verbreitung dieses für Chikungunya- und Dengue-Fieber verantwortlichen primären Vektors zu rechnen. Chikungunya ist charakterisiert durch hohes Fieber, Myalgien, Exantheme und starke Arthralgien, was Chikungunya von Dengue unterscheidet [11]. Des Weiteren wird erwartet, dass heisse Sommer das Vorkommen und die Verbreitungsdynamik von Zecken verändern und damit zu einer Ausbreitung der zeckenübertragenen Krankheiten FSME (Frühsommer-Meningoenzephalitis) und Borreliose beitragen [12]. In Regionen mit guten Gesundheitssystemen und solidem Umweltmanagement wird der Klimawandel für das Vorkommen der - einst auch in der Schweiz heimischen - Malaria hingegen vernachlässigbar sein. Die durch Sandmücken übertragene Leishmaniose ist in Europa endemisch und birgt bei wärmeren Temperaturen ein gewisses Risiko der zunehmenden Verbreitung. Leishmaniose kann sich durch ulzerierende Läsionen der Haut oder Schleimhäute manifestieren [11].

$\mathrm{Zu}$ betonen ist, dass das Auftreten und die Verbreitung Vektor-übertragener Infektionskrankheiten in der Schweiz nicht primär durch den Klimawandel, sondern vielmehr durch das Reiseverhalten der Bevölkerung bestimmt werden.

Das Auftreten von Nahrungsmittel- und Wasser-bedingten Infektionskrankheiten nimmt während Hitzeperioden ebenfalls zu. Ein entscheidender Faktor für das Auftreten von derartigen Infektionskrankheiten sind der unsachgemässe Umgang mit Lebensmitteln sowie fehlende Hygienemassnahmen [13].

Des Weiteren kann die Zunahme der Durchschnittstemperatur die Pollensaison verlängern und das Auftreten neuer Allergene begünstigen (z.B. von legal und illegal importierten Neophyten), was eine zunehmende Belastung für Allergiker darstellt.

Hitzebedingte Gesundheitsrisiken haben komplexe Mechanismen und werden durch eine Vielzahl von Faktoren (sozioökonomische, ökologische usw.) beeinflusst. Sie stellen eine grosse Herausforderung für das Gesundheitssystem dar.

Der Klimawandel ist nicht nur eine lokale Herausforderung, sondern stellt vielmehr ein globales Problem dar. Die im fünften Sachstandbericht der IPCC (Intergovernmental panel on climate change) 2014 veröffentlichten Ergebnisse verdeutlichen die grossen globalen Herausforderungen in der Klimapolitik: Die reichen Länder des Westens, die in den letzten Jahrhunderten die Hauptverursacher des Klimawandels waren, sind zunehmend in der Lage, durch lokale Massnahmen die direkten Folgen des Klimawandels einzudämmen. Einkommensschwache Länder im Süden sind hingegen nicht nur viel stärker vom Klimawandel betroffen, sondern verfügen kaum über genügende Ressourcen für die Umsetzung gezielter Vorsorgemassnahmen. Somit werden die Folgen des Klimawandels in diesen Ländern viel grösser sein als bei uns. Dadurch wird sich der Migrationsdruck in reichere Länder erhöhen [14].

Die diesjährige Konferenz von Public Health Schweiz widmet sich in Genf den Chancen und Risiken des Klimawandels und insbesondere den Auswirkungen der Klimapolitik auf die Gesundheit der Bevölkerung. Das Haupt thema der Konferenz lautet in diesem Jahr «Public Health Benefits of Climate Change Policies». Nationale und internationale Expertinnen und Experten werden nicht nur die Auswirkungen des Klimawandels auf die Gesundheit aufzeigen, sondern auch den indirekten gesundheitlichen Nutzen von Klimaschutzmassnahmen für die Gesundheit der Bevölkerung diskutieren.

Die Swiss Public Health Conference findet vom 17. bis 18. September 2015 am Institut de Santé Globale der Universität Genf statt. Anmeldung: http://conference. public-health.ch

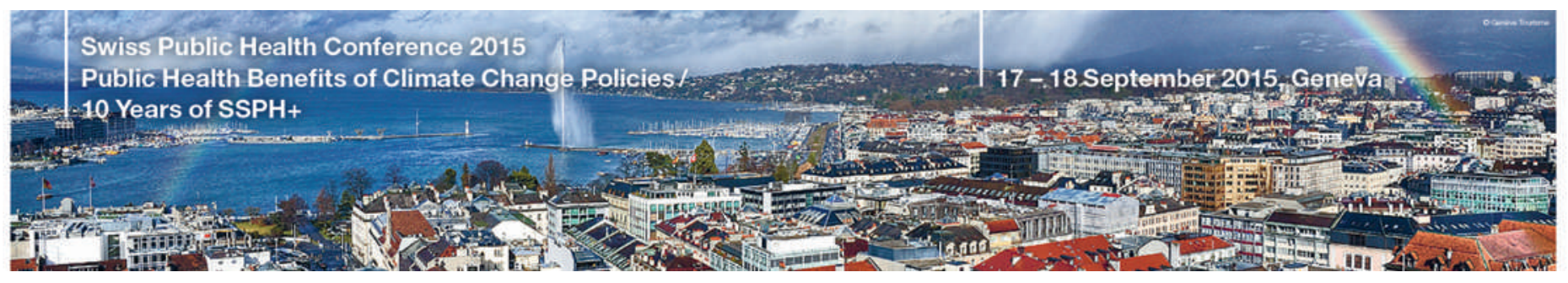




\section{Literatur}

1 Costello A, Abbas M, Allen A, Ball S, Bell S, Bellamy R, et al. Managing the health effects of climate change: Lancet and University College London Institute for Global Health Commission. Lancet. 2009;373:1693-733.

2 OcCC Beratendes Organ für Fragen der Klimaänderung. Hitzesommer 2003, Synthesebericht. Bern, November 2005.

3 Robine JM, Cheung SLK, Le Roy S, Van Oyen H, Griffiths C, Michel JP, et al. Death toll exceeded 70,000 in Europe during the summer of 2003. Comptes Rendus Biologies. 2008;331:171-8.

4 Grize L, Huss A, Thommen O, Schindler C, Braun-Fabrlander C. Heat wave 2003 and mortality in switzerland. Swiss Med Wkly. 2005;135:200-5.

5 Thommen Dombois O, Braun-Fahrländer C. Gesundheitliche Auswirkungen der Klimaänderung mit Relevanz für die Schweiz. Literaturstudie im Auftrag der Bundesämter für Umwelt, Wald und Landschaft (BUWAL) und für Gesundheit (BAG). 2004.

6 Gronlund CJ, Zanobetti A, Schwartz JD, Wellenius GA, O'Neill MS. Heat, heat waves, and hospital admissions among the elderly in the United States, 1992-2006. Environ Health Perspect. 2014;122:1187-92.

7 Michelozzi P, Accetta G, De Sario M, D'Ippoliti D, Marino C, Baccin $M$, et al. High temperature and hospitalizations for cardiovascular and respiratory causes in 12 European cities. American Journal of Respiratory and Critical Care Medicine. 2009;179:383-9.

8 Bundesamt für Gesundheit (BAG). Informationsmaterial und Verhaltensempfehlungen verfügbar unter: www.hitzewelle.ch
9 Fouillet A, Rey G, Wagner V, Laaidi K, Empereur-Bissonnet P, Le Tertre A, et al. Has the impact of heat waves on mortality changed in France since the European heat wave of summer 2003 ? A study of the 2006 heat wave. Int J Epidemiol 2008;37(2):309-17.

10 ECDC (European Centre for Disease Prevention and Control). Climate change and communicable diseases in the EU Member States, Handbook for national vulnerability, impact and adaptation assessments. 2010

11 Bassetti S. "Neue» Infektionskrankheiten in der Schweiz durch den Klimawandel? Schweiz Med Forum. 2009;9(50):905

12 Gray JS, Dautel H, Estrada-Peña A, Kahl O, Lindgren E. Effects of Climate Change on Ticks and Tick-Borne Diseases in Europe. Interdiscip Perspect Infect Dis. 2009:593232.

13 Bless JP, Schmutz C, Suter K, Jost M, Hattendorf J, MäusezahlFeuz $\mathrm{M}$ et al. A tradition and an epidemic: Determinants of the campylobacteriosis winter peak in Switzerland. European Journal of Epidemiology. 2014; 29(7):527-37.

14 IPCC, 2014: Climate Change 2014: Impacts, Adaptation, and Vulnerability. Part B: Regional Aspects. Contribution of Working Group II to the Fifth Assessment Report of the Intergovernmental Panel on Climate Change. Barros, V.R., C.B. Field, D.J. Dokken, M.D. Mastrandrea, K.J. Mach, T.E. Bilir, M. Chatterjee, K.L. Ebi, Y.O. Estrada, R.C. Genova, B. Girma, E.S. Kissel, A.N. Levy, S. MacCracken, P.R. Mastrandrea, and L.L. White (eds.). Cambridge University Press, Cambridge, United Kingdom and New York, NY, USA, $688 \mathrm{pp}$. 